\title{
A study on the bandwidth characteristics of pleated pneumatic artificial muscles
}

\author{
Rino Versluys ${ }^{\mathrm{a} *}$, Kristel Deckers $^{\mathrm{b}}$, Michaël Van Damme ${ }^{\mathrm{a}}$, Ronald Van Ham ${ }^{\mathrm{a}}$, Gunther Steenackers ${ }^{\mathrm{b}}$, Patrick Guillaume ${ }^{\mathrm{b}}$ \\ and Dirk Lefeber ${ }^{\mathrm{a}}$ \\ ${ }^{a}$ Robotics and Multibody Mechanics Research Group, Vrije Universiteit Brussel, Brussels, Belgium; \\ ${ }^{b}$ Acoustics and Vibration Research Group, Vrije Universiteit Brussel, Brussels, Belgium
}

(Received 23 December 2008; final version received 27 January 2009)

\begin{abstract}
Pleated pneumatic artificial muscles have interesting properties that can be of considerable significance in robotics and automation. With a view to the potential use of pleated pneumatic artificial muscles as actuators for a fatigue test bench (high forces and small displacements), the bandwidth characteristics of a muscle-valve system were investigated. Bandwidth is commonly used for linear systems, as the Bode plot is independent of the amplitude of the input signal. However, due to the non-linear behaviour of pleated pneumatic artificial muscles, the system's gain becomes dependent on the amplitude of the input sine wave. As a result, only one Bode plot is insufficient to clearly describe or identify a non-linear system. In this study, the bandwidth of a muscle-valve system was assessed from two perspectives: a varying amplitude and a varying offset of the input sine wave. A brief introduction to pneumatic artificial muscles is given. The concept of pleated pneumatic artificial muscles is explained. Furthermore, the different test methods and experimental results are presented.
\end{abstract}

\section{Introduction}

Pneumatic technology is largely used in factory floor automation. To date, there exist a large range of pneumatic actuators. A less known type is that of the pneumatic artificial muscle (PAM). This actuator is comparable with an inverse bellows, as it shortens when inflated. The actuator's pulling force is, besides the gauge pressure, also dependent on the contraction level, which creates a source of springlike behaviour. Pneumatic muscles can transfer the same amount of energy as other pneumatic actuators do, since they operate at the same pressure ranges and volumes. PAMs are extremely lightweight, as they are basically no more than a membrane. Moreover, they have some additional benefits, such as easy replacement, direct connection to the system's structure, safe operation and no risk of fire and explosion.

McKibben developed a type of PAM for use as an orthotic actuator in the late 1950s. The McKibben muscle (Schulte 1961) seemed to be an ideal choice for this purpose, but several drawbacks, such as complexity of control, less sophisticated valve technology at that time, (pneumatic) power conservation and availability, drastically decreased the interest in this actuator. Although the use of electric drives meant that pneumatic technology was largely discontinued - especially in prosthetics and orthotics - there was continued work on specialised actuators. Advanced computing techniques combined with improvements in control strategies and the realisation that for many robotic applications the performance of electric drives and in general their rather low power-to-weight ratio was inadequate prompted new interest in actuator design, and many advantages associated with pneumatic technology have been revisited.

Daerden and Lefeber (2001) developed the pleated pneumatic artificial muscle (PPAM), a new design of PAM. It has been shown that this design is an improvement in comparison with the braided muscle regarding several actuator characteristics. The PPAM was used as an actuator for the first time in the bipedal walking robot Lucy (Vanderborght et al. 2005). Thanks to its compliant behaviour, it has another broad field of interest: assistive technology in non-industrial domains, like health care (Versluys et al. 2008).

The principle aim of this study was to investigate whether a PPAM carries potential as an actuator for a fatigue test bench. Current fatigue test benches use hydraulic actuators, which have significant disadvantages such as high weight of the actuator, high cost, oil leakage, high frequency of maintenance and the complexity of the hydraulic servo control. We decided to choose PPAMs above pneumatic cylinders because of their very high force-to-weight ratio, low cost and the fact that they do not require any maintenance. Since the response behaviour of the actuator (i.e. a PPAM) plays a dominant role in fatigue testing, we have examined the PPAM's bandwidth in the $0 \%$ to $4 \%$ contraction region (small displacements, high forces). A major part in modelling pneumatic muscles has been done by Caldwell et al. (1995), Chou and Hannaford (1996), Klute

*Corresponding author. Email: rino.versluys@vub.ac.be

This work was supported by the FWO Flanders, Belgium under Grant G.0496.06.a

ISSN: $1176-2322$ print / 1754-2103 online

Copyright (C) 2009 Taylor \& Francis

DOI: $10.1080 / 11762320902738647$

http://www.informaworld.com 


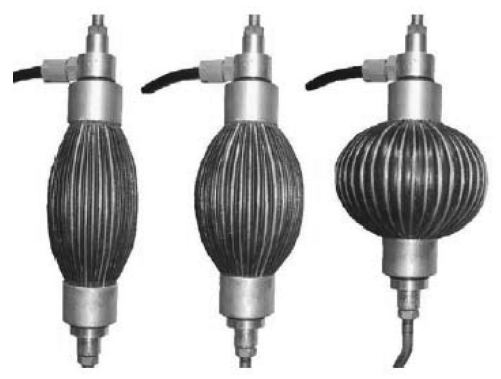

Figure 1. PPAM at three contraction levels. (This figure is available in colour online.)

and Hannaford (1998) and Tsagarakis and Caldwell (2000). The bandwidth of pneumatic muscles has previously been studied by Davis et al. (2002). The aforementioned publications describe the measurement, the modelling and the dynamic assessment of the McKibben muscle. In the current study, however, the bandwidth characteristics of PPAMs are assessed. As a result, the presented results are new, and we believe they can be of high importance for further research projects in which the PPAM fulfills a crucial role and for pneumatic muscle users in general. This paper is organised as follows: in Section 2 a short introduction to PPAMs and a comparison with conventional pneumatic cylinders is given; Section 3 reports on the methodology and results of the performed experiments. In Section 4, a summary and some recommendations are given.

\section{Pleated pneumatic artificial muscles}

\subsection{Concept and operation}

A photograph of a PPAM at three contraction levels is given in Figure 1. The core element of a PPAM is a pleated reinforced membrane, attached at both ends to fittings, along which mechanical power is transferred to a load. As the volume, enclosed by the membrane, is inflated or deflated, the device bulges out or elongates, respectively. Related to this radial expansion or compression, the artificial muscle contracts or stretches axially and exerts a pulling force on its load. The PPAM's energy source is compressed air. That way the actuator is powered by the pressure difference between the internal air and the ambient air. Because of the unfolding effect, there is no threshold pressure; contractions of up to $40 \%$ are possible; and hysteresis is reduced.

Bearing in mind the low weight of the PPAM, its high power-to-weight ratio and inherent compliant behaviour, it is clearly understood that this actuator carries a lot of potential to replace conventional actuators in factory floor automation and many other mechatronical applications. What follows next is a brief comparison between a conventional pneumatic cylinder and a PPAM of quasi-similar dimensions. A pneumatic cylinder weighing $240 \mathrm{gm}$ with a length of $15 \mathrm{~cm}$ and a diameter of $2.5 \mathrm{~cm}$ can generate a force of $275 \mathrm{~N}$ at a pressure of $6 \mathrm{bar}$ (http://www.festo.com). A PPAM weighing $140 \mathrm{gm}$ with a length of $11 \mathrm{~cm}$ and a

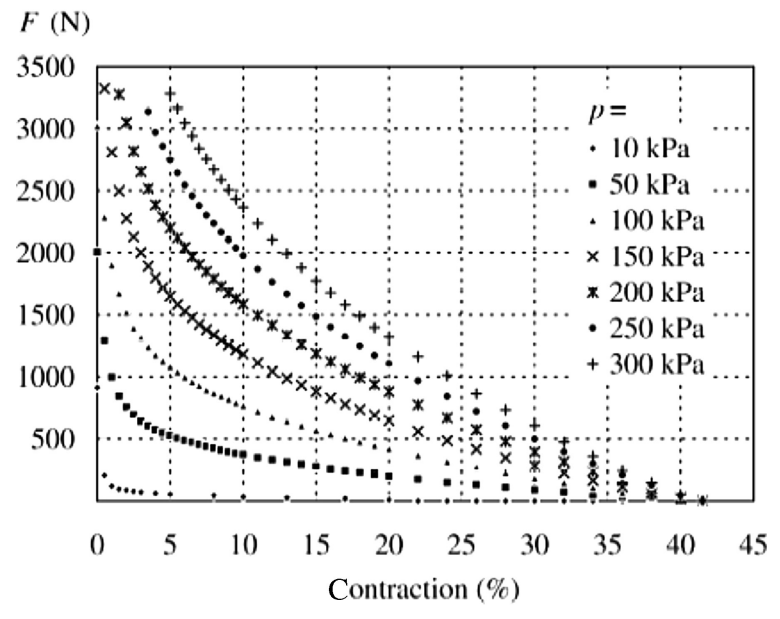

Figure 2. Generated force versus contraction level.

minimum diameter of $3 \mathrm{~cm}$ can generate a force of 4500 $\mathrm{N}$ at a pressure of only 3 bar. Thus, a PPAM with these dimensions generates forces that are 16 times higher (at half the pressure) than a pneumatic cylinder with similar dimensions.

\subsection{Characteristics}

The pulling force $F$, exerted by the PPAM, can be set as

$$
F=p \cdot L^{2} \cdot f\left(\varepsilon, \frac{L}{R}, a\right)
$$

in which $p$ represents the internal air pressure in the PPAM, $L$ its full length, $\varepsilon$ its contraction, $R$ its minimum radius and $a$ a dimensionless factor which takes account of the membrane's elasticity. If this membrane consists of material with high tensile strength and stiffness, the influence of elasticity can be dropped and set to zero. As a result, $f$ is a non-linear, dimensionless function that depends on contraction rate and geometry (expressed by the slenderness $L / R$ ). The forcecontraction curves of a PPAM with a diameter of $3 \mathrm{~cm}$ and a length of $11 \mathrm{~cm}$ are shown in Figure 2. When investigating the potential of a PPAM as actuator for a fatigue test bench, we are interested in high forces and small displacements. $\mathrm{We}$, therefore, investigated the dynamic response only in the $0 \%$ to $4 \%$ contraction region, in which the forces are of significant magnitude.

\section{System bandwidth}

\subsection{Introduction}

As mentioned earlier, the concept bandwidth is commonly used for linear systems, as the Bode plot is independent of the amplitude of the applied sinusoidal signal. However, due to the nonlinear behaviour of a PPAM, the system's gain becomes dependent on the amplitude of the sinusoidal input signal. As a result, only one Bode plot is insufficient to clearly describe or identify a non-linear system. 


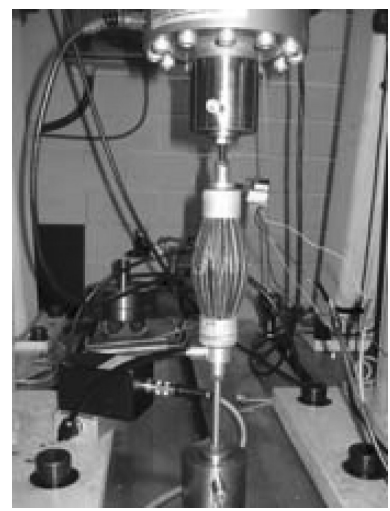

Figure 3. Experimental set-up. (This figure is available in colour online.)

In this study, the dynamic response of a PPAM-valve system was investigated from two perspectives; varying amplitude and varying offset of the applied sinusoidal input signal. This way, the influence of both the amplitude and the offset of the applied signal on the system's bandwidth could be investigated.

\subsection{Methodology}

A PPAM (length $=11 \mathrm{~cm}$, diameter $=3 \mathrm{~cm}$, volume $=300$ $\mathrm{ml}$ ) was at its one end attached to a steel frame. The other end was screwed into the (fixed) piston of an INSTRON ${ }^{\circledR}$ hydraulic test bench for dynamic and fatigue tests (see Figure 3).

Consider Figure 4. The experimental set-up consists of a PPAM, a KPS $3 / 4-10^{\circledR}$ servo valve (Kolvenbach, Germany) with a maximum mass flow of $550 \mathrm{Nl} / \mathrm{min}$ at a pressure difference of $6 \mathrm{bar}$, and a pressure vessel serving as compressed air supply. We used a load cell and a BSDX5000G2 ${ }^{\circledR}$ pressure sensor (Sensortechnics, Germany), attached to an end fitting of the PPAM for muscle pressure information. PC 1 runs a MATLAB stack and sends the desired input sine wave (in volts) to the servo valve. The servo valve makes use of its built-in proportional-integral-derivative (PID) device to control the output pressure. An increment of $1 \mathrm{~V}$ of the input signal corresponds to an increment of 1 bar of the output pressure. PC 2 communicates with the control unit of the test bench to keep the hydraulic piston fixed and to make sure that the force does not exeed $3500 \mathrm{~N}$, which is the force limit due to the material properties of the PPAM.

First, to calculate the static gain, we excited the PPAM with a sinusoidal signal having a frequency of $0.1 \mathrm{~Hz}$ and an amplitude of 0.5 bar, oscillating around an offset of 1.1 bar (see Figure 5).

The first set of experiments was performed with the PPAM kept at quasi-zero contraction. Both the offset and the amplitude of the sinusoidal input signal were varied in order to clearly identify the bandwidth of the PPAM-valve system.

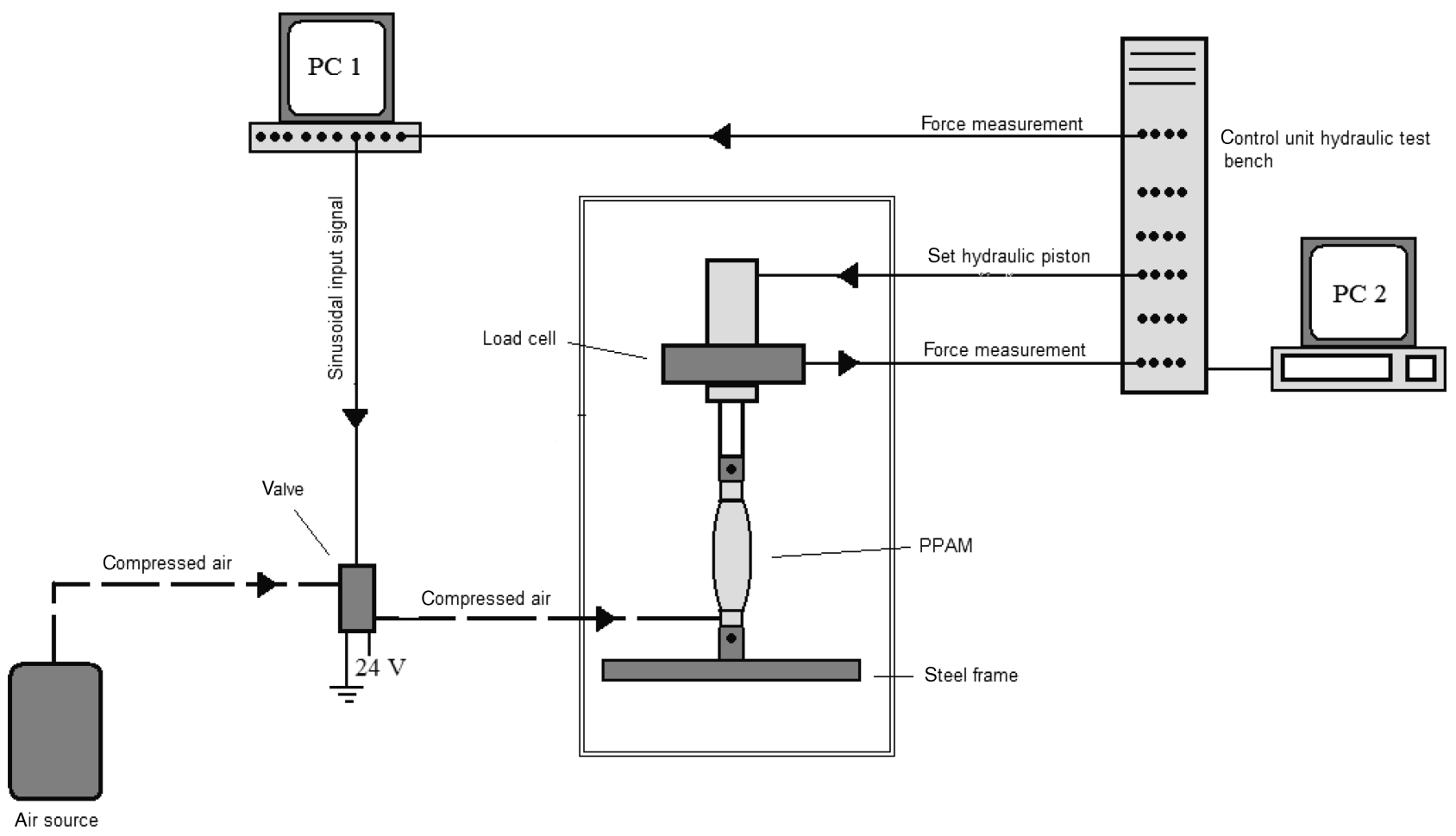

Figure 4. Schematic representation of the experimental set-up. 
- Force $(\mathrm{kN})$

— Input signal $(\mathrm{V})$

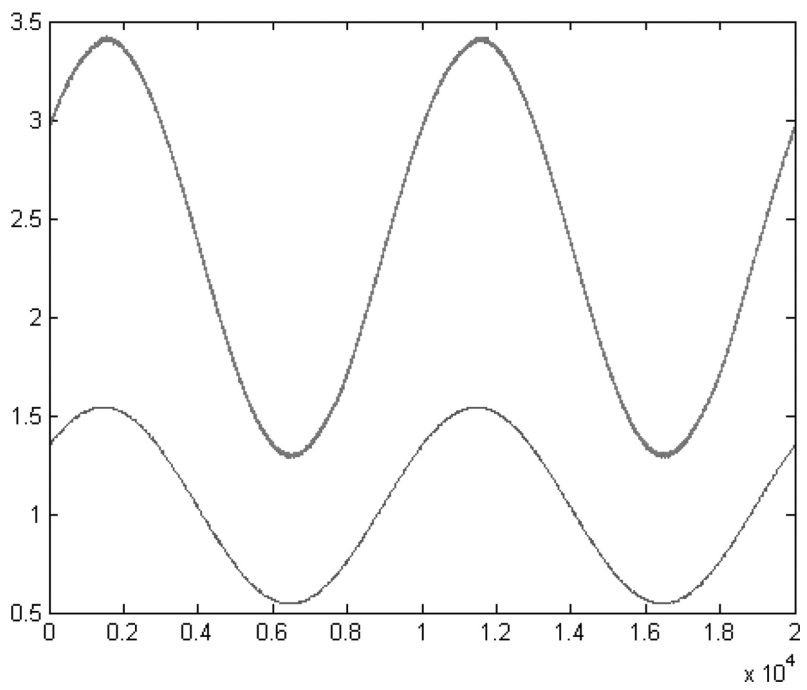

Figure 5. Calculating the static gain. (This figure is available in colour online.)

In a second set of experiments, the contraction level of the PPAM was varied between $0 \%$ and $4 \%$ contraction to investigate the influence of contraction on the system's bandwidth.

After calculating the static gain we explored the frequency range from $1 \mathrm{~Hz}$ up to $15 \mathrm{~Hz}$ and modified the amplitude or the offset of the applied sinusoidal signal.

The next section gives an overview of some experimental results. Since many tests were performed, only the most relevant results are shown.

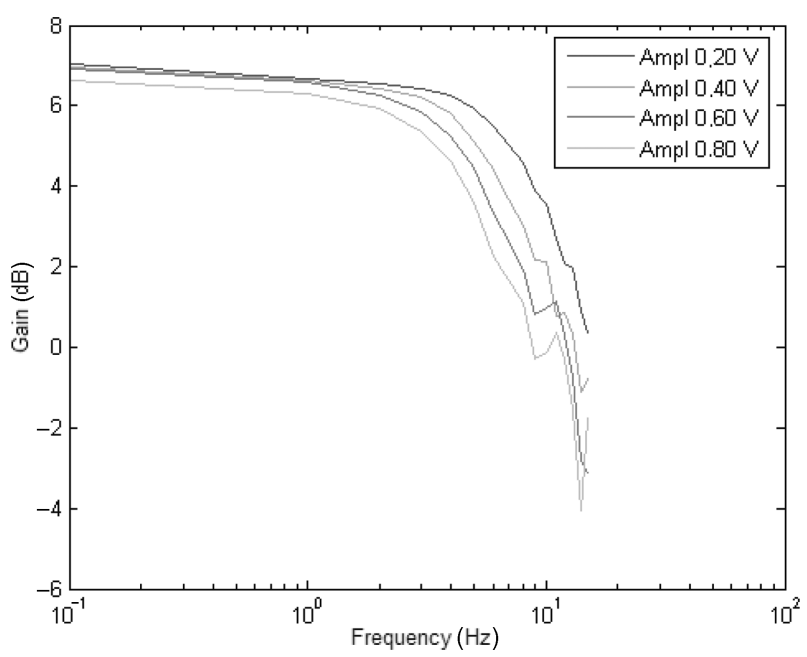

Figure 6. Bode plots for constant pressure offset of 0.8 bar and varying pressure amplitude. (This figure is available in colour online.)

\subsection{Experimental results and discussion}

\subsubsection{At (quasi-)zero contraction}

Figure 6 shows different Bode plots, extracted from the experimental data. A constant signal was sent to the pneumatic valve in order to obtain a constant pressure $(0.8$ bar $)$ in the PPAM.

The PPAM valve system was excited with sinusoidal signals of varying pressure amplitudes (see the legend in Figure $6 ; 1 \mathrm{~V}$ corresponds to $1 \mathrm{bar}$ ). The maximum allowed input value was derived at zero contraction and comes to 1.6 bar. As a result, the maximum applied voltage may not exceed $1.6 \mathrm{~V}$ with respect to the upper force limit of $3500 \mathrm{~N}$.

With regard to the amplitude variations at a constant pressure offset, one can see the lowering of the Bode plots as the amplitude increases (Figure 6). This means that, as the pressure amplitude increases at constant pressure offset, the corresponding increase of the generated force reduces so that the gain lowers. This effect is due to the non-linear behaviour of the system.

Figure 7 depicts the bandwidth of the PPAM valve system for varying pressure amplitude and a constant pressure offset of 0.8 bar. The bandwidth decreases with higher pressure amplitudes. We attribute this effect to the inherent inertia of the pneumatic system. The higher the muscle pressure (higher amplitude), the higher the mass flow needs to be. Yet, due to the valve flow limits of the Kolvenbach ${ }^{\circledR}$ KPS $3 / 4$ valve, the bandwidth will decrease as the mass flow increases.

Within a second experiment, we kept the amplitude of the sinusoidal input signal constant and varied the pressure offset values from 0.5 bar up to $1.1 \mathrm{bar}(1 \mathrm{~V}$ corresponds to 1 bar). The results are shown in Figure 8.

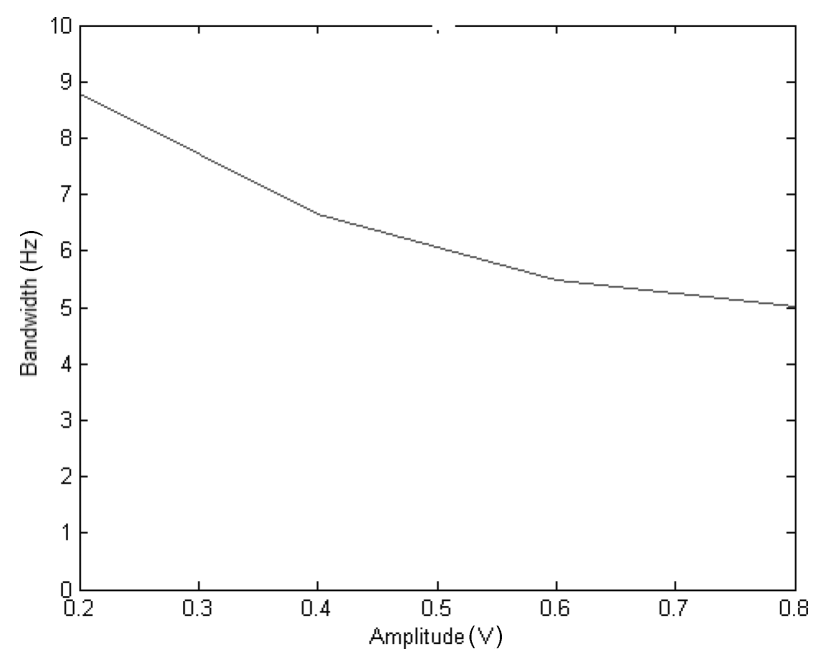

Figure 7. Bandwidth of the PPAM-valve system for varying pressure amplitude. (This figure is available in colour online.) 


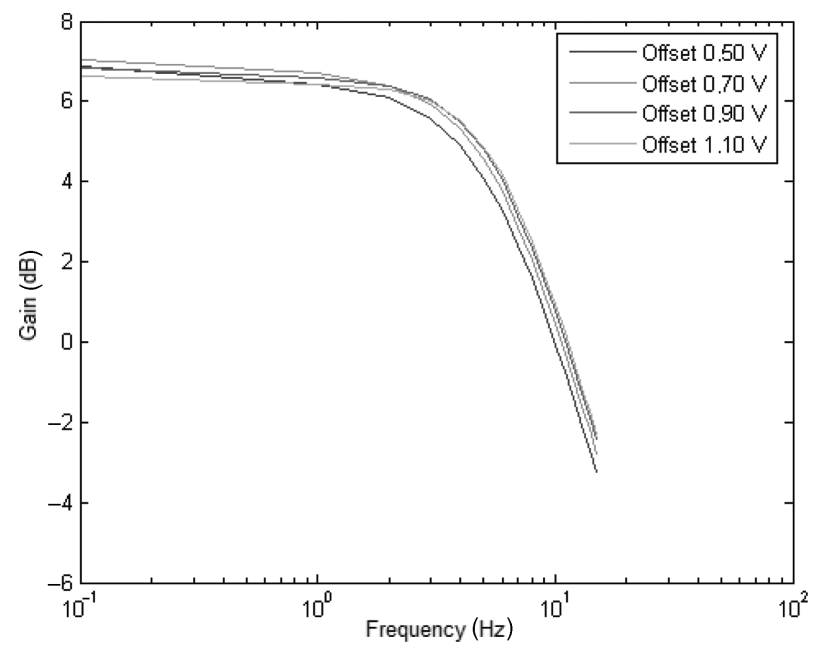

Figure 8. Bode plots for constant pressure amplitude and varying pressure offset. (This figure is available in colour online.)

All Bode plots nearly coincide for the whole frequency range. This potentially indicates that the non-linear effects are rather independent of the applied offset. Because the Bode plots nearly coincide it is self-evident that the corresponding bandwidth values are almost similar. This can be seen in Figure 9.

\subsubsection{At different contraction levels}

The previous experiments at zero contraction give a first view on the frequency behaviour of the PPAM-valve system but do not take into account the influence of the contraction level of the PPAM. To cope with this we performed some experiments with the PPAM being fixed at certain contraction levels. The maximum contraction of the PPAM was limited

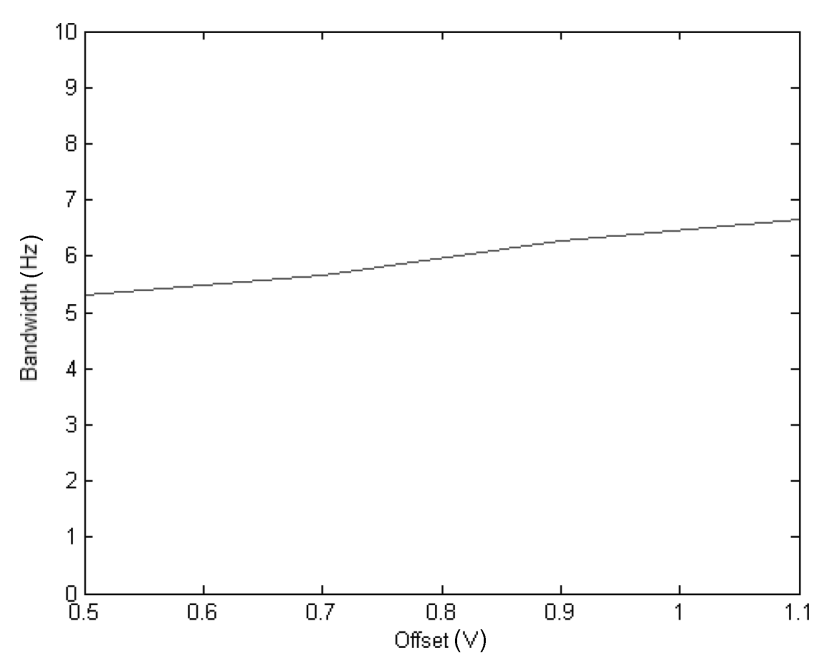

Figure 9. Bandwidth of the PPAM-valve system for varying pressure offset. (This figure is available in colour online.)

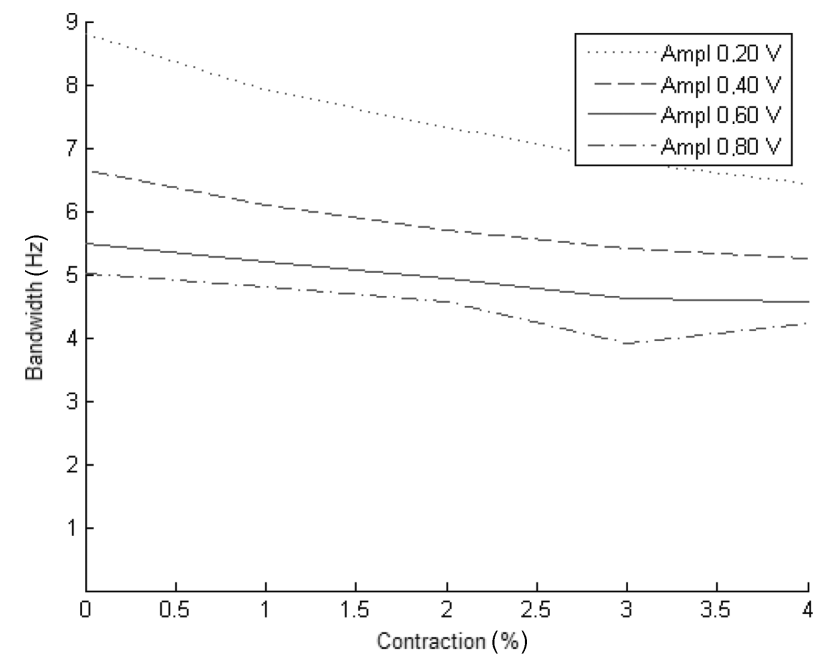

Figure 10. Bandwidth of the PPAM versus contraction level (varying pressure amplitude). (This figure is available in colour online.)

to $4 \%$, as higher contractions were of non-significant use regarding the corresponding lower forces (fatigue test bench $\Rightarrow$ high forces and low displacements). The practical set-up is similar to that shown in Figure 4. Figure 10 depicts the influence of the contraction level on the bandwidth. Similar to the experiments at zero contraction, a constant pressure was applied to the PPAM-valve system with the PPAM being at a certain contraction level. The system was excited with sinusoidal signals of different pressure amplitude (see legend in Figure 10;1 V corresponds to 1 bar). One can notice that the bandwidth decreases as the contraction becomes higher, independent of the amplitude. The decrease is lower at higher amplitudes.

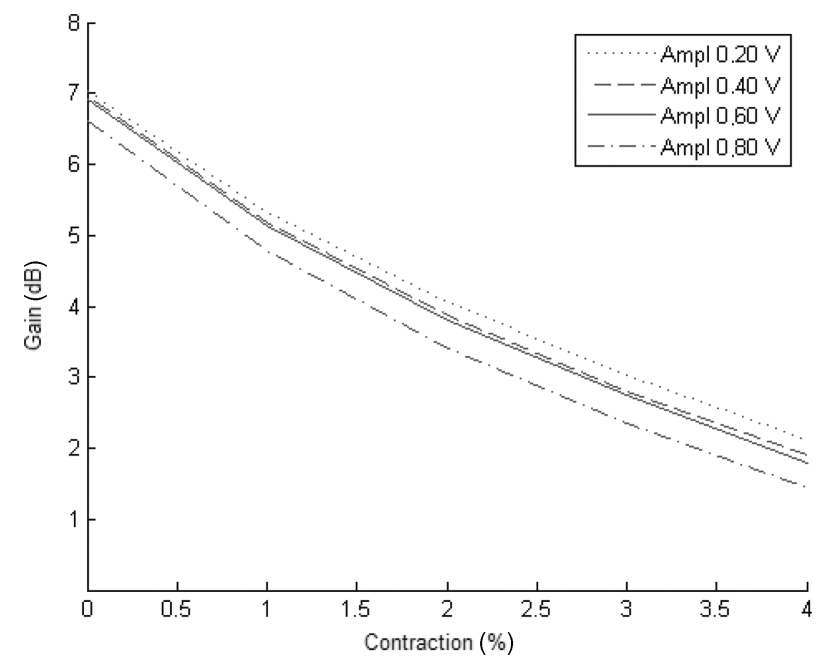

Figure 11. Gain versus contraction level (varying amplitude). (This figure is available in colour online.) 


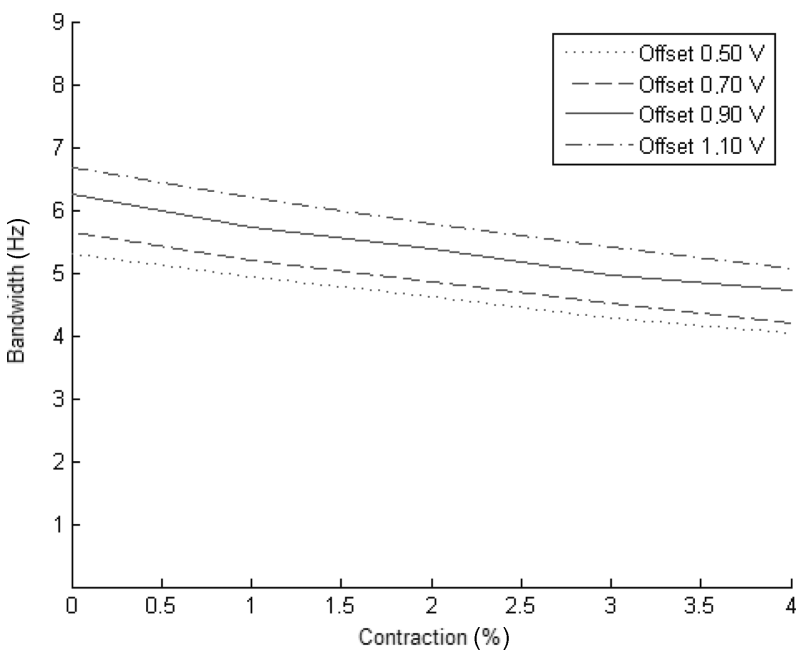

Figure 12. Bode plots for varying pressure offset. (This figure is available in colour online.)

This decrease in bandwidth with a higher contraction can be potentially attributed to the (rather small) increase in muscle volume as the contraction increases. As a result, the mass flow needs to be greater. The higher the volume of the PPAM, the lower the dynamic response. This was earlier reported by Davis et al. (2002).

The same effect appears as during the zero contraction experiments, namely the decreasing bandwidth with higher amplitudes. This effect was attributed to the inertia of the PPAM-valve system.

Figure 11 deals with the variation of the gain as a function of the contraction, at constant offset and varying amplitude.

Figure 12 represents the influence of the pressure offset on the bandwidth of the PPAM-valve system. The pressure

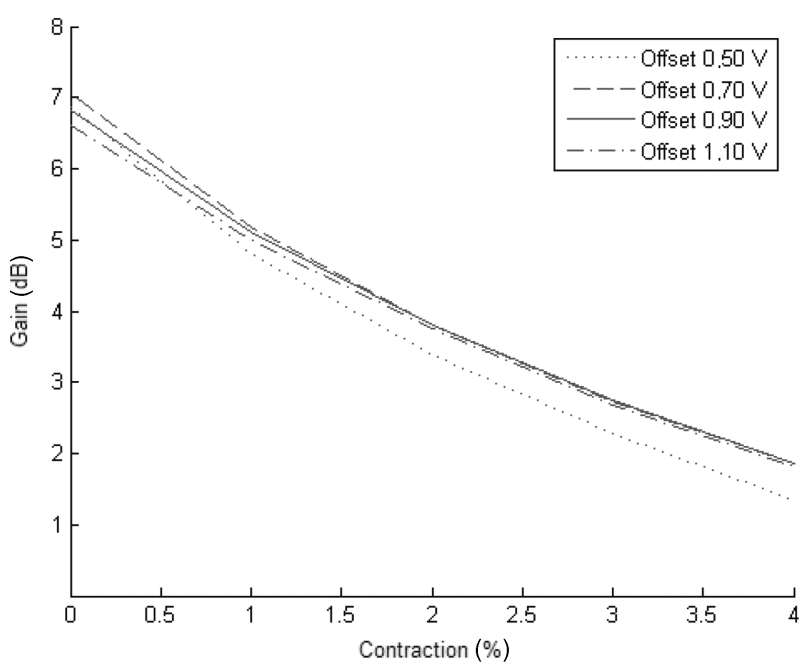

Figure 13. Gain versus contraction level (varying offset). (This figure is available in colour online.)
Table 1. Overview of the bandwidth of the PPAM-valve system.

\begin{tabular}{lcr}
\hline & $\begin{array}{c}\text { Bandwidth at } \\
\text { zero contraction }\end{array}$ & $\begin{array}{r}\text { Bandwidth at } \\
4 \% \text { contraction }\end{array}$ \\
\hline Varying amplitude & $5-9 \mathrm{~Hz}$ & $\begin{array}{r}4.5-6.5 \mathrm{~Hz} \\
4-5.5 \mathrm{~Hz}\end{array}$ \\
Varying offset & $5-6.5 \mathrm{~Hz}$ & \\
\hline
\end{tabular}

offset was varied from 0.5 bar to $1.1 \mathrm{bar}$ ( $1 \mathrm{~V}$ corresponds to $1 \mathrm{bar}$ ), while the amplitude of the sinusoidal input signal was kept constant ( 0.5 bar). In general, the bandwidth lowers as the contraction increases. The slope of the curves is the same for each offset. A reduction in bandwidth of about 1.5 $\mathrm{Hz}$ is noted.

Figure 13 shows the relationship between the gain and the contraction, at a frequency of $0.1 \mathrm{~Hz}$. The force reduction is nearly equal for the whole amplitude range. The reduction touches $5 \mathrm{~dB}$ at the $4 \%$ contraction level. As the contraction increases, the gain decreases. One can see that the influence of the offset is rather small, since the different curves nearly coincide.

\section{Summary and recommendations}

Table 1 gives an overview of the different experimental results.

During this study, we investigated the dynamic response of a PPAM-valve system in order to examine the potential use of a PPAM as actuator for a pneumatic fatigue test bench. As shown in Table 1, bandwidth values of about 6 $\mathrm{Hz}$ at $4 \%$ contraction level and $9 \mathrm{~Hz}$ at zero contraction level were achieved. Although the performed experiments were still preliminary and situated within the framework of a greater study, we believe that the results are promising. It is, however, not easy to predict whether a pneumatic fatigue test bench will counterbalance the conventional test benches. Nevertheless, the advantages of a pneumatic fatigue test bench are the low cost (compressor, valves and pneumatic muscles), the simplicity and the easy transportation of the pneumatic set-up.

We investigated the bandwidth of the PPAM-valve system using a PPAM of specific length and diameter. Of course, the influence of the muscle dimensions needs to be investigated, since it has been shown that the bandwidth increases with smaller volumes (Davis et al. 2002).

Also the length of the pneumatic cables and the capacity of the compressed air source will both have an influence on the dynamic response.

Furthermore, the maximum mass flow of the servo valve is for sure one of the most dominant parameters influencing the system's bandwidth.

Therefore other experiments such as filling the muscle with grains to reduce the volume, shortening the supply cables and using other valves are the subject of further research. 


\section{References}

Caldwell DG, Medrano-Cerda GA, Goodwin MJP. 1995. Control of pneumatic muscle actuators. IEEE Control Syst. 15(2.2):40-48.

Chou P, Hannaford B. 1996. Measurement and modeling of McKibben pneumatic artificial muscles. IEEE Trans Rob Automat. 12(2.2):90-102.

Daerden F, Lefeber D. 2001. The concept and design of pleated pneumatic artificial muscles. Int J Fluid Power 2(3):41-50.

Davis S, Canderle J, Artrit P, Tsagarakis N, Caldwell DG. 2002. Enhanced dynamic performance in pneumatic muscle actuators. Proceedings of the 2002 IEEE International Conference on Robotics and Automation. Washington DC, USA. p. 28362841.

Klute GK, Hannaford B. 1998. Fatigue characteristics of McKibben artificial muscle actuators. Proceedings of the 1998 IEEE International Conference on Intelligent Robots and Systems. Victoria, Canada. p. 1776-1781.
Schulte HF. 1961. The characteristics of the McKibben artificial muscle. In: The application of external power in prosthetics and orthotics. National Academy of Sciences National Research Council. p. 94115.

Tsagarakis N, Caldwell DG. 2000. Improved modelling and assessment of pneumatic muscle actuators. Proceedings of the 2000 IEEE International Conference on Robotics and Automation. San Francisco, USA. p. 3641-3646.

Vanderborght B, Verrelst B, Van Ham R, Van Damme M, Versluys R, Lefeber D. 2005. The pneumatic biped 'LUCY' actuated with pleated pneumatic artificial muscles. Auton Robots 18:201-213.

Versluys R, Desomer A, Lenaerts G, Pareit O, Vanderborght B, Van der Perre G, Peeraer L, Lefeber D. 2008. A biomechatronical transtibial prosthesis powered by pleated pneumatic artificial muscles. Int J Modelling Identification Control 4(4):394405. 

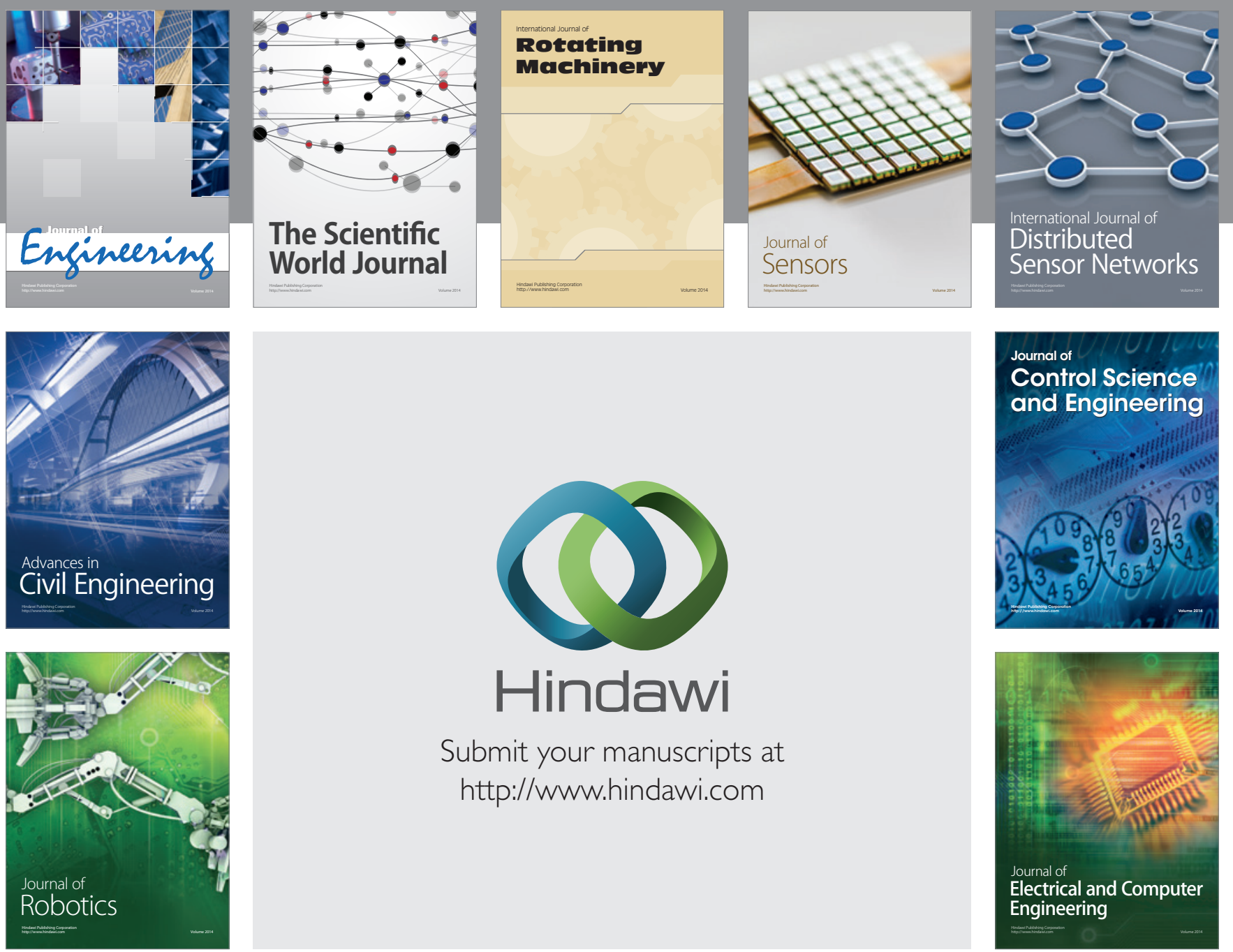

Submit your manuscripts at

http://www.hindawi.com
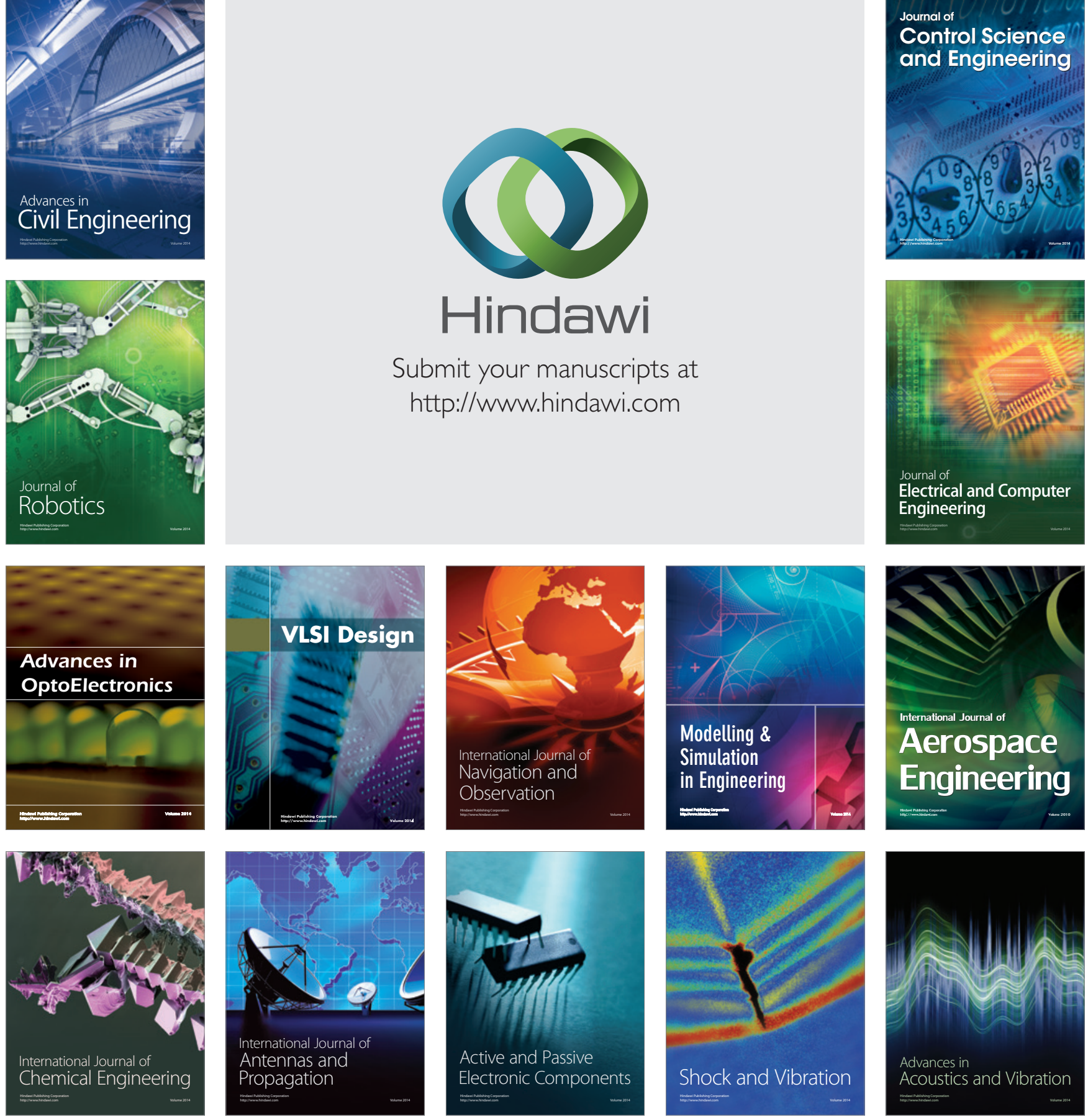